\title{
Spectinomycin Hydrochloride
}

National Cancer Institute

\section{Source}

National Cancer Institute. Spectinomycin Hydrochloride. NCI Thesaurus. Code C47728.

The hydrochloride salt form of spectinomycin, an aminocyclitol antibiotic derived from Streptomyces spectabilis with antibacterial property. Spectinomycin hydrochloride binds to the bacterial 30 S ribosomal subunit, thereby inhibiting peptide elong ation and protein synthesis, consequently leading to bacterial cell death. 